\title{
The Development of a Learner Model in Adaptive Hypermedia Educational Systems: A Comparative Study
}

\author{
Mouenis Anouar Tadlaoui ${ }^{1}$, Rommel N. Carvalho ${ }^{2}$, Mohamed Khaldi ${ }^{1}$ \\ ${ }^{1}$ LIROSA, Faculty of Sciences, Abdelmalek Essaâdi University, Tétouan, Morocco \\ ${ }^{2}$ University of Brasília, Department of Computer Science, Brasilia, Brazil
}

\begin{abstract}
It's worth noting that the present paper lies within the range of modeling the learner in adaptive educational system as a conceptual modeling of the learner. The learner model is an essential component for Adaptive eLearning systems. The term adaptation in e-Learning systems involves the selection and manner of presentation of each learning activity as a function that examines the entity of knowledge, skills and other information given by each subject taught. The present paper aims at studding the functionalities of the learner model in different Adaptive Hypermedia Educational Systems in the three stages of developing and managing this model. We will present in this comparative study, a full analysis of the learner model used in ten major hypermedias to come up with most appropriate method to treat the dynamic aspect of this model.
\end{abstract}

\section{Introduction}

In general, the adaptation process can be described in three steps: retrieving user information, processing information to initialize the user model, and updating the initialized user model; finally, the use of the user model to provide the adaptation. In this chapter, we will only use the user's model term, which is used because the user profile is simply considered the process of collecting raw data from the user. [1]

In the process of adaptation, it is possible to distinguish between two different characters. At the beginning, the learner or student with his goal of acquiring knowledge, second: the teacher. The goal of a teacher is to mediate the knowledge covered by a course to learners. Therefore, both points of view must be present in an e-Learning system. [2]

To be able to adapt the presentation of learning and navigation content to the needs of the user, a user model is needed, including objectives or tasks, knowledge, prerequisites and user preferences. These user properties are used to make adaptive decisions by adaptive hypermedia systems. In addition, the user's recent templates also store the interests and individual traits.

In this paper, we will begin by presenting the learner model in Adaptive Hypermedia Educational Systems (AHES), its main role and categories that will form the context of this paper. The focus then will shift to the study of the main functionalities of the learner model. We will divide these functionalities into six categories and we will present a study in 10 different hypermedias to conclude in which functionalities they're based. Then, we will put emphasis on the different phases to develop and model the learner model in adaptive educational hypermedia systems and illustrate the range of application of each functionalities and aspect and it focus area. We aim in this paper at presenting a comparative study of each aspect and functionalities of the learner model in different adaptive educational systems, and at showing their level/degree of intervention in relation with the components of the learner model and development process.

\section{Learner model in Adaptive Hypermedia educational systems}

In this section, we will present the categories of a learner model in adaptive hypermedia educational systems. And then will present the main functionalities of the learner model, and a comparative analysis of their existence in 10 existing hypermedia.

\subsection{The categories of a learner model in AHES}

Today's AHES are putting more and more emphasis on the intelligence of the system. One of the most important factors in assessing the quality and usability of the system is the level to meet the needs of the user, the learner. So the learner model, the component that backs up and manages learner information, becomes more important.

A Learner model is one of the major components of an AHES. It allows to keep information about the learner, for example his level of knowledge on a given subject (performance), his frequent errors / misunderstandings, his psychological characteristics, etc. A learner model can be defined as a set of structured information about the learning process, and this structure contains values about the characteristics of the learner. It provides the necessary data for the other modules in order to adapt the teaching to the learner [3], [4]. 


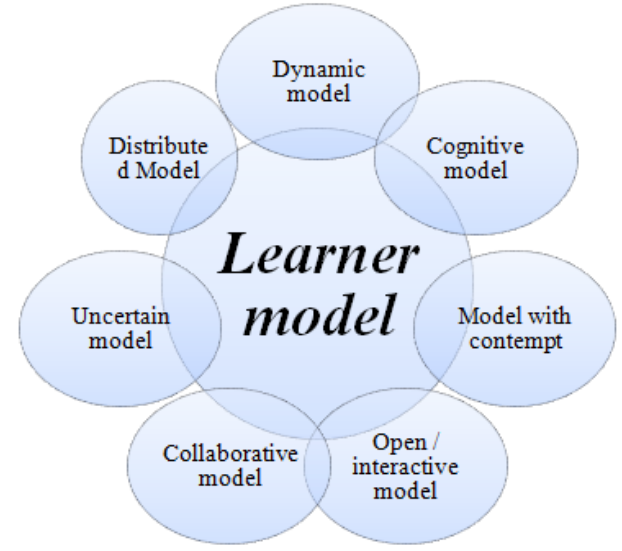

Figure 1. the categories of a Learner model in AHES

Figure 1 represents the different categories of a learner model existing in different AHES. Each learner model must belong to one or more of these main categories:

Dynamic Model: This category of learner model accumulates and records in real time the information about the learner. This information could be modified or updated dynamically depending on the interaction between the learner and the system, e.g.: system visits, answers to questions, etc. As a result, the system actively adapts the teaching to the learners; in particular it supports the dynamic navigation in quantity of educational materials.

Cognitive model: The cognitive model means that the cognitive aspects of the learner are considered in modeling. For example, the principles of cognitive psychology are adapted and incorporated in the modeling, to follow the state of the memory of the learner, in order to adapt pedagogical strategies to the needs of the learner among others by offering him relevant contents to be revised at the appropriate time. Many researchers have applied cognitive theories to modeling in a variety of ways. In addition, cognitive characteristics of the learner, for example, the attitude of learning, the time that a fact remains in the memory, the capacity of memory, are frequently included in this category of a learner model.

Model with contempt: In this category, the learner model mainly retains the errors or the frequent misunderstandings of the learners and also the causes or explanations of these errors. Normally, this list of errors is a result of empirical studies involving many students and tutors in concrete cases in schools. This type of model is often used to simulate a learner's problemsolving process to diagnose possible errors. In addition, the misunderstanding model could be used for the automatic construction of exercises, for example in the case of multiple-choice questions, to automatically produce the most common erroneous answers given by students.

Open / Interactive Model: This new approach to learner modeling allows learners to have some control over diagnostic inspection and model modification. The model is built jointly by the system and the learner. The system then accumulates its own points of view and those of the learner on the knowledge of the learner. The open model is used to encourage learners to reflect on their learning, as well as for tutors to tailor instruction to the individual or group. The openness of the model could also make it easier for learners to compare their own progress over time or their own progress with peers in the same group or other groups.

Collaborative model: This model is used in the context of collaborative learning. Collaborative learning is a new paradigm that broadens a classical AHES by involving concepts of collaboration. The success of a student could help the success of other members of the group. The learner could also search for an online peer, the system that matches students according to relevant attributes, to solve a problem. The collaborative model is also useful for finding peers who could or would like to receive feedback about some aspects of their work.

Uncertain Model: This category of learner model focuses on dealing with the uncertainty of the learner's knowledge. Descriptions of knowledge or levels of knowledge are often inaccurate in the actual situation. Knowledge modeling can represent, train and update this uncertain knowledge. The treatment of uncertainty could also help to reach certain conclusions about learner knowledge or pedagogical strategies, based on incomplete information.

The Distributed Model: This learner model is often used in distributed environment. There is no monolithic model associated with each student. Knowledge about a specific student is distributed among agents who communicate with that student. Modeling is a process of assembling and summarizing fragmented learner information from potentially diverse sources. This information could be raw data recorded by an application, partially calculated models, and learner options recorded by a tutor or peers, or a history of the learner's actions.

We briefly presented the characteristics and usefulness of the different categories of learner model. It is difficult to attribute rigid and definitive boundaries to these categories. Some concrete learner models have several characteristics, that is, they belong to several different categories. We could find in some situations, the same learner model in some AHES is in the same time a dynamic, distributed and collaborative model. To determine the most appropriate learner model category, one needs to thoroughly analyze the practical needs and real situations. And to achieve this purpose, we will try to conduct this comparative study with 10 different AHES.

\subsection{The existing Adaptive Hypermedia Educational Systems}

In this study to comprehend the main functionalities of a learner model in adaptive hypermedia educational 
systems, we have chosen 10 AHES from different domains of intervention. We will compare each one of them based on some well-defined metrics to define a proper path to construct and initialize a learner model using a combination of methods/model.

- $\boldsymbol{A H A}$ !, the "Adaptive Hypermedia Architecture", was originally developed to support an on-line course with some user guidance through conditional (extra) explanations and conditional link hiding. It consists of a set of concepts, some of which are linked to pages or objects (or fragments). Concepts can be used to represent topics of the application domain [5].

- The ADAPTWEB environment is an adaptive hypermedia system providing the same content adapted to different students' groups. ADAPTWEB is an open source environment in operation in different universities [6].

- $\boldsymbol{A} \boldsymbol{V A N T I}$ is a system designed for a range of users with different needs. This system combines in the stages of data collection and initialization of the learner model stereotypes and superposition method to create initial assumptions, and to maintain the knowledge of the user [7].

- ANATOM-TUTOR Developed for teaching anatomy of the brain at university level, ANATOM-TUTOR's hypertext component makes use of a user model to adapt hypertext lessons at both the link and the text level. Hypertext is one of three learning modes offered by ANATOM-TUTOR [8].

- The $\boldsymbol{A} \boldsymbol{H} \boldsymbol{M}$ system, in which the adaptation depends on the level of expertise on the concepts of the field know the system is a subset of all the user's domain concepts [9].

- The $\boldsymbol{E L M} \boldsymbol{A} \boldsymbol{A R T}$ system is an Adaptive distance Tutor system, which support learning Lisp programming language. It uses different techniques for initializing and updating its learning model: overlay model, the complex machine learning methods and Bayesian networks to represent episodic learning model [10].

- INSPIRE is an adaptive hypermedia that emphasizes the fact that learners perceive and process information in very different ways and integrates ideas from theories of instructional design and learning styles. INSPIRE, throughout its interaction with the learner, dynamically generates learner-tailored lessons that gradually lead to the accomplishment of learner's learning goals [11].

- In HYPERADAPT, a specialized approach utilizing an aspect-oriented programming is used. This hypermedia places the adaptivity into separate modules called adaptation aspects. The aspects are not applied on a model level, but on XML documents [12].

- HYNECOS is adaptive hypermedia that demonstrates the applicability of the Hypertext Design Model HDM for design of hypertext-based information systems from relational databases. HYNECOS contains textual and graphical data about patients (administrative data, reports, X-rays etc.), hospital staff (names, telephones and shift-information about all staff members), a medical encyclopedia (diseases, treatments, and prognosis) and the location of the wards (room-plans, beds, occupancy etc.) [13].

- The METADOC system is an adaptive hypermedia mainly used in medicine, it's not only has hypertext capabilities but also has knowledge about the documents it represents. This knowledge enables the document to modify its level of presentation to suit the user. METADOC builds and dynamically maintains a user model for each reader [14].

\subsection{The functionalities of a learner model in existing AHES}

The intelligence of an AHES is mainly attributed to its ability to adapt to a specific learner during the teaching process; adaptation depends on the individual learner's knowledge of the subject taught and other relevant characteristics of the learner; relevant knowledge and information of the learner is often managed in a learner model [15], [16], [17].

The learner model category plays an important role in the implementation of intelligence and personalization of AHES. Specifically, a learner model can help personalize learning, assess learner knowledge, track learner progress, etc.

In Table 1, we identified six essential functionalities of a learner model: Personalizing learning, assessment of the learner's knowledge, follow-up and presentation of progress and actions of the learner, search for appropriate peers to get help, management of the learner information and automatic construction of exercises. And we will compare the existence of these functionalities in the AHES we took into our comparative study.

According to this table, we can conclude that two features are very common. Systems provide personalized learning by adapting teaching to the needs of the learner, giving relevant advice. Planning own learning activities, etc. Appropriate exams or tests are built according to the learner's performance. 
Table 1. Comparative table between the functionalities of a learner model in existing AHES

\begin{tabular}{|c|c|c|c|c|c|c|c|c|c|c|}
\hline The functionalities of a learner model & 这 & 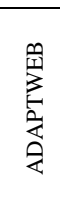 & 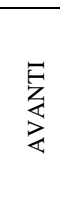 & 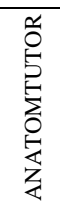 & 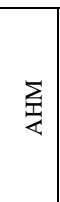 & $\begin{array}{l}\frac{1}{2} \\
\frac{1}{2} \\
\tilde{Z}\end{array}$ & 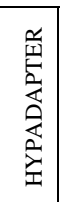 & 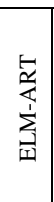 & 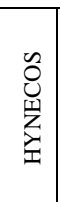 & $\begin{array}{l}\text { ○ } \\
\text { 空 } \\
\stackrel{5}{\Sigma}\end{array}$ \\
\hline Personalizing learning & $\mathrm{X}$ & - & $\mathrm{X}$ & $\mathrm{X}$ & $\mathrm{X}$ & $\mathrm{X}$ & - & $\mathrm{X}$ & - & - \\
\hline Adaptation of teaching & $\mathrm{X}$ & $\mathrm{X}$ & $\mathrm{X}$ & - & - & - & - & - & - & - \\
\hline Adapted presentation of teaching materials & $\mathrm{X}$ & - & - & $\mathrm{X}$ & - & $\mathrm{X}$ & - & - & - & - \\
\hline Planning and organization of activities or educational contents & - & - & - & $\mathrm{X}$ & - & $\mathrm{X}$ & - & - & - & - \\
\hline Selection of relevant teaching methods / strategies & - & - & $\mathrm{X}$ & - & - & - & - & $\mathrm{X}$ & - & - \\
\hline Personalized help / advice & - & - & - & - & - & - & - & $\mathrm{X}$ & $\mathrm{X}$ & $\mathrm{X}$ \\
\hline Adaptive assessment & - & $\mathrm{X}$ & - & - & $\mathrm{X}$ & - & - & - & - & - \\
\hline Planning learning activities & $\mathrm{X}$ & - & $\mathrm{X}$ & - & - & - & - & - & - & - \\
\hline Adaptive navigation & $\mathrm{X}$ & $\mathrm{X}$ & - & - & - & - & $\mathrm{X}$ & $\mathrm{X}$ & - & - \\
\hline Assessment of the learner's knowledge & - & - & - & $\mathrm{X}$ & $\mathrm{X}$ & - & - & - & $\mathrm{X}$ & $\mathrm{X}$ \\
\hline Analyzes of learner responses / beliefs & $\mathrm{X}$ & - & $\mathrm{X}$ & - & $\mathrm{X}$ & - & - & - & - & - \\
\hline Diagnosing the mistakes of the learner & - & - & $\mathrm{X}$ & $\mathrm{X}$ & $\mathrm{X}$ & - & - & - & - & - \\
\hline Planning the diagnostic dialogue & - & - & $\mathrm{X}$ & & $\mathrm{X}$ & $\mathrm{X}$ & - & - & - & - \\
\hline Adaptive explanations & $\mathrm{X}$ & - & & $\mathrm{X}$ & - & - & - & - & - & - \\
\hline Resolving ambiguities of error explanations & $\mathrm{X}$ & - & $\mathrm{X}$ & - & - & - & - & - & - & - \\
\hline Follow-up and/or presentation of progress and actions of the learner & - & $\mathrm{X}$ & - & - & - & - & $\mathrm{X}$ & - & $\mathrm{X}$ & - \\
\hline Search for appropriate peers to get help & - & & - & - & $\mathrm{X}$ & - & - & - & $\mathrm{X}$ & - \\
\hline Management of the learner information & - & $\mathrm{X}$ & - & - & $\mathrm{X}$ & - & $\mathrm{X}$ & - & $\mathrm{X}$ & $\mathrm{X}$ \\
\hline Automatic construction of exercises & - & $\mathrm{X}$ & - & - & $\mathrm{X}$ & - & $\mathrm{X}$ & & $\mathrm{X}$ & $\mathrm{X}$ \\
\hline
\end{tabular}

In addition, adaptive navigation has long been a technique used to achieve adaptation of learning. More specifically, to adapt teaching, teaching materials can be presented according to the level of knowledge and according to the learner's personal preference. Educational planning can also be organized according to the learner's knowledge, psychological characteristics or preferences. Pedagogical strategies can be selected according to the psychological characteristics and the learner's performance.

Learner model is also an essential component in assessing the learner's knowledge. For example, learner responses can be analyzed to infer beliefs, in other words, correct, erroneous, or incomplete knowledge. We can diagnose errors or misunderstandings of the learner according to the frequent mistakes of this learner and those of many students. Information on these errors is maintained in the learner model. Students' knowledge can be accessed from a conversation between the system and the learner. This diagnostic dialogue can be planned according to the learner's beliefs. From the results of the diagnosis, the explanations of the errors are adapted to each student according to his. In some cases, when there are ambiguities about the explanations, the learner's error history can be consulted to determine the appropriate explanations.

The other functionalities are also important and interesting even if they are present in few systems, for example: the monitoring of the progress of the learner, the automatic construction of exercises, etc. The presentation and comparison of the student's progress encourages the student to become more active. The automatic (or semi-automatic) construction of exercises allows tutors to increase their effectiveness. Peer research is very useful in collaborative models.
The majority of systems have two or more features at the same time, especially the two essential features of personalization of learning and assessment of learner knowledge, for example, AHM, AHA!, ANATOMTUTOR, etc. Some systems focus on a single feature, which presents a mechanism for representing and maintaining the learner's actions by noting temporal aspects, in order to provide the learner with an overview of his or her model (long-term and short-term). This feature is important for getting relevant learner information and can be useful for other features, including learning adaptation and ambiguities resolution in diagnosing learner errors or misunderstandings.

\subsection{The composition of a Learner model in existing AHES}

Based on the survey we have presented in our previous work [20], we could resume the composition of learner models in different AHES into four major categories: characteristics of the learner, learning state, Learner Knowledge and Interactions between the system and the learner. [18]

- Characteristics of the learner: This category includes the learner's general or psychological characteristics, such as his or her learning purpose, type of learning, learning style preferences, computer experiences, level of learning concentration, the desired level of detail, availability, etc. The level of concentration could be inferred according to its interactions with the system, for example the time it is no longer active in the system. From this information, we can specify the anticipated needs of the learner.

- Learning state: Current or past states are classified in this category. More specifically, this type of model 
contains the learning plan, the program followed, the learning history, etc. This information is then used to analyze and maintain the learner's situation.

- Interactions between the system and the learner: This is one of the key categories of learner information. Interactions between the system and the learner are recorded and updated. At the appropriate time, the system infers the learner's knowledge or learning status according to the recorded data. These interactions can be divided into two sub-categories: system visits and answers to questions. Visits include visits to educational content, such as the number of visits to the same unit, the duration of the same visit, the type of content, etc. The number of examples or aids requested is also recorded. Then the answers include the correct answers and the wrong answers. In fact, the system does not keep the answers; instead it records the corresponding parameters, for example the number of errors, the frequency of a particular error, the more frequent errors, the number of tests, etc.

- Learner Knowledge: This is another important category. The data or information in the previous categories is used to infer the learner's knowledge to provide a personalized learning. In this category, there may be the learner's level of knowledge, exam or test scores, chess patterns, learner's beliefs and degrees of correction, explanations of errors/misunderstandings, knowledge of prerequisite concepts, knowledge of sub-domains, knowledge to be validated, knowledge acquired, etc.

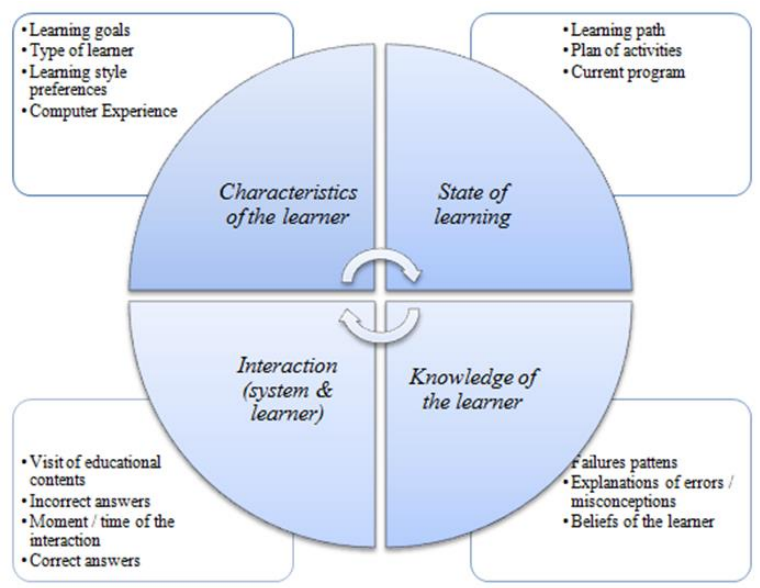

Figure 2. The composition of a Learner model in AHES

Figure 2 shows the proportion of the use of each category in AHES. It is impossible and not necessary to build a complete learner model with all the elements mentioned, because it requires too many resources. Most of the proposed learner models contain only a few parts of these four categories, especially the last two. Although researchers place less emphasis on learning status, it is useful for personalizing learning by considering the learner's learning path and progress.

\section{Development of a learner model in Adaptive hypermedia educational systems}

After presenting the functionalities of the learner model in different AHES. We will present in this section of the paper, the three phases of learner model's development in AHES. We will begin by explaining the process of learner model development and then we will present a comparative study concerning the three phases of the development process according to 10 AHES.

\subsection{The process of developing a learner model in AHES}

Despite these various attempts to model the learning that is characterized by a dynamic aspect, we always find it difficult to achieve this goal. The proposed approaches give us just a static view of the learner model, but in practice this model is in full development (the learner knowledge is evolving in the same module) to a dynamic view. In order to monitor the behavior of the learner in real time during training, we must adopt a model for dynamic management of the learning model.

All actions of the learner in a learning situation are not limited to valid or invalid actions (true and false), but these actions are characterized according to the learner learning path during his training. From this observation, we cannot represent the information from the system of each learner as a relative data. This demand putting our work in a probabilistic context due to the changes in the learner model during training. [19]

Figure 3 represents the stages of development of a model of the learner. In the data collection phase, they are three major steps: the collection of data about the user, the collection of data about the system and creating a learner profile based on this initial data collected.

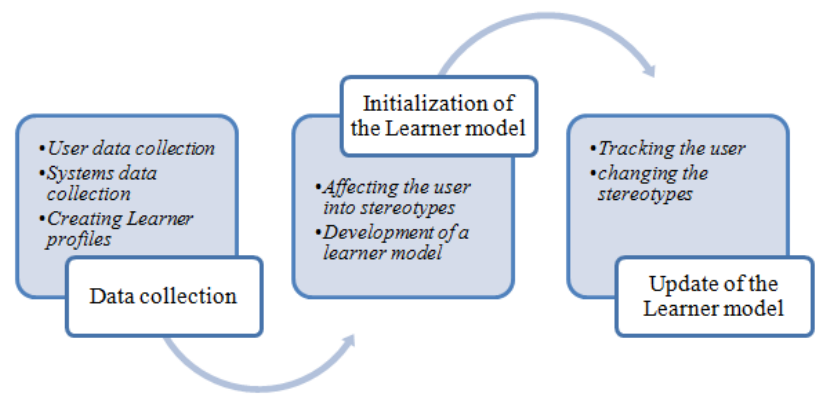

Figure 3. The process of developing a learner model in AHES

In the initialization stage of a learner model, the collected data undertakes a transformation process towards the model of the learner. This initialization process is realized through the implementation of several methods. The system affects the user to a specific stereotype according to the data collected. Then continue to collect data about the user all along his learning path using several methods like Bayesian networks or machine learning. 
The information that is used to update the learner model can be retrieved, implicitly or explicitly from various sources of information. Among the sources of information, we find the information currently stored in the learner model. This information can be used as a source for which we derive new information or make changes to the alleged information. Furthermore, the information currently stored in the other system components may be useful.

\subsection{The construction of a learner model in existing AHES}

Table 2 represents a comparative between these different aspects of the construction of a learner model in AHES. We could notice that mainly all the AHES dispose of a system for data composition and representation.

Table 2. Comparative table between the different aspect of the constraction of a learner model in existing AHES

\begin{tabular}{|c|c|c|c|c|c|c|c|c|c|c|}
\hline $\begin{array}{l}\text { Construction of the learner model (data } \\
\text { collection) }\end{array}$ & 至 & 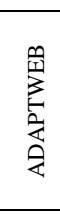 & 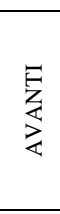 & 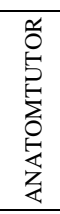 & 要 & 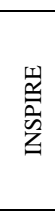 & 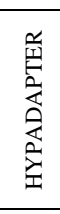 & 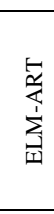 & 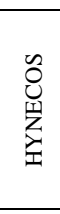 & 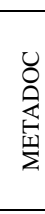 \\
\hline Data Composition / structure & - & $\mathrm{X}$ & $\mathrm{X}$ & $\mathrm{X}$ & $\mathrm{X}$ & $\mathrm{X}$ & $\mathrm{X}$ & - & - & $\mathrm{X}$ \\
\hline Data representation & $\mathrm{X}$ & $\mathrm{X}$ & $\mathrm{X}$ & $\mathrm{X}$ & $\mathrm{X}$ & $\mathrm{X}$ & - & - & $\mathrm{X}$ & $\mathrm{X}$ \\
\hline Data collection general construction methods & $\mathrm{X}$ & - & $\mathrm{X}$ & - & $\mathrm{X}$ & $\mathrm{X}$ & - & $\mathrm{X}$ & - & $\mathrm{X}$ \\
\hline Long-term / short-term model of data collection & - & - & - & - & $\mathrm{X}$ & - & $\mathrm{X}$ & - & - & - \\
\hline Group / individual model of data collection & - & - & - & $\mathrm{X}$ & - & - & - & $\mathrm{X}$ & $\mathrm{X}$ & - \\
\hline Empirical studies & - & $\mathrm{X}$ & - & - & - & - & - & - & $\mathrm{X}$ & - \\
\hline
\end{tabular}

The main difference between this AHES is the methods used to collect data; we could notice that the old AHES based its data collection in general construction methods, and the specific AHES used more individual methods to extract precise data about the user.

The construction of an MA concerns the determination of the composition and structure of a learner model. For example, what categories of information should be included in the learner model? What concrete elements are needed? And what are the links of these elements between them? We must also consider how to effectively represent this structure and these elements. Some Hypermedia concretely presents the use of certain formalisms of representation. The process or method of construction indicates how to organize and acquire this information and knowledge. For example, how to extract knowledge from empirical studies.

\subsection{The inisialisation of a learner model in existing AHES}

The initialization of a learner model is a fairly important step in the development process. Researchers have exploited four ways to initialize a learner model: through questionnaires or pre-tests, by stereotypes, by the previous cases and by the default values. Among them, stereotypes are most often used. Stereotypes represent the knowledge and characteristics of typical user classes. So each student is assigned to one of the predefined classes (i.e.: stereotypes). The major task of initialization is to select the appropriate stereotype for a learner. The questionnaires are practical and relatively easy to implement. Therefore, they are used in several systems.

Table 3 represents a comparative between these different aspects of the initialization of a learner model in AHES.

Table 3. Comparative table between the different aspect of the initialization of a learner model in existing AHES

\begin{tabular}{|c|c|c|c|c|c|c|c|c|c|c|}
\hline The initialization of the learner model & 起 & 离 & $\begin{array}{l}\overrightarrow{\mathrm{z}} \\
\overrightarrow{\mathrm{x}} \\
\mathrm{z}\end{array}$ & 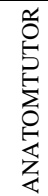 & 要 & 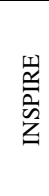 & 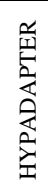 & 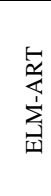 & 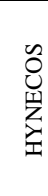 & 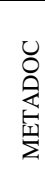 \\
\hline Pre-tests / questionnaires & - & $\mathrm{X}$ & $\mathrm{X}$ & - & - & - & - & $\mathrm{X}$ & - & - \\
\hline Previous cases & $\mathrm{X}$ & - & - & - & - & - & $\mathrm{X}$ & - & - & - \\
\hline Stereotypes & - & - & $\mathrm{X}$ & $\mathrm{X}$ & - & $\mathrm{X}$ & - & - & $\mathrm{X}$ & $\mathrm{X}$ \\
\hline By default & - & - & - & - & - & - & - & - & - & $\mathrm{X}$ \\
\hline Machine learning & - & $\mathrm{X}$ & - & - & $\mathrm{X}$ & $\mathrm{X}$ & - & $\mathrm{X}$ & - & - \\
\hline
\end{tabular}


Sometimes, to know the initial level of knowledge of the learner, the learner is asked to do a simple test before starting his apprenticeship. The result of the test could be used to initialize some learner model values, or to show the progress of learning.

Using previous cases to initialize the model is an interesting method. Previous experiences (models) of individual students or groups are used as stereotypes for future learners. Default values are used frequently in traditional systems. The initial values are assigned and then adjusted as appropriate in the treatment process.

\subsection{The update and management of a learner model in existing AHES}

The methods and steps of a learner model management are related to the appropriate times to update the data or knowledge stored in the models. The learner model maintenance mechanism is specific to everyone because it has a close relationship with many aspects of the model, such as its structure, mode of representation, use, and so on.

Table 4 represents a comparative between these different aspects of the update and management of a learner model in AHES.

Some parameters in the category of personal data are used to identify the learner, for example the name, the user name or login name, the email, etc. Some personal data (eg age, experiences, gender, training, etc.) and some characteristics of the learner (learning purpose, type / style of learning, computer experiences, level concentration, preferences, etc.) are used to initialize the model, especially to select the appropriate stereotype for the learner. A stereotype represents the common characteristics of the learning style. Learning paths or activity plans could also be exploited to preserve the present or past states of the learner The statistical parameters concerning the interaction between the learner and the system (the frequency of a specific error, the units visited more frequently, etc.) are the results of the calculation or reasoning based on certain visible parameters on the actions of the learner. the learner. They could also change some characteristics of the learner, including the level of concentration. Interaction parameters and characteristics are used to evaluate the learner's knowledge. Conversely, the level of knowledge assessed could also influence / modify certain characteristics of the learner.

\section{The modeling approaches used for learner modelling in existing AHES}

A learner model is a combination of all relevant data on the learner in relation to a learning environment. There are common types of information among learners' models such as object domain information, goals, motivation, training and experience, cognitive skills, preferences and demographic training data.

To develop and manage the learner model, several methods/techniques have been used. Table 5 represents a comparative between these different modeling techniques and models used in modelling the learner in AHES.

\section{Discussion}

Learner models can be used in very different ways, depending on the actions and characteristics of the learner in the system. Since there are different types of adaptive e-Learning systems, the applied learner models are different.

In many systems, the learner model may not be explicitly described as a simple functional module. It can be spread over several elements of the system. Thus, it is clearly visible what is connected to the model of the learner. Therefore, a learner model may not be available as an additional component but properties, which are related to a user model and are assigned to the long-term model user.

We could say, based on this study, that the learner model is an essential element in adaptive e-Learning systems. The adaptation of an e-Learning system primarily involves the selection and presentation of each successive teaching activity based on the full scope of the learner's knowledge of the subject taught and other relevant characteristics of the subject. As a result, the learner model is used to change the interaction between the system and the student to meet the individual needs of the students.

A learner model must be built, initialized and updated. Initializing a learner model is an important topic, where an appropriate way to gather the requested information has to be found. In particular, the effort for the user during initialization should be considered as this process affects the accuracy and usability of the learner model and the entire system. To keep the information stored on the learner updated, the modification of learner information must be included in the learner template. After the information within a learner's model is changed, the new information must be delivered. Delivery affects systems that use the learner model and should keep information consistent across all places where it is used.

\section{Conclusion}

An adaptive system needs information about the target to which it adapts. Since this target is most often adaptive to the learner of the adaptive system, a learner model is required. By using a learner model, an adaptive system can use this learner model in three different types of system actions. The system can interpret the actions of the learner differently regarding the features stored in the learner's model. For example, users with dyslexia may have a particular problem with a few words. The system recognizes these typos and automatically corrects the entry. In the other direction, the actions of the machine or the output of the system can be adapted to meet the needs of the user, and finally, internal actions can be influenced by the information stored in the user's model. 
Table 4. Comparative table between the different aspect of the update and use of a learner model in existing AHES

\begin{tabular}{|c|c|c|c|c|c|c|c|c|c|c|}
\hline $\begin{array}{c}\text { The update and management of the learner } \\
\text { model }\end{array}$ & 袁 & 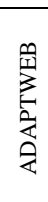 & $\underset{4}{3}$ & 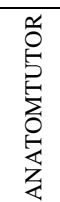 & $\sum_{\frac{1}{4}}$ & 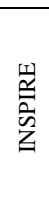 & 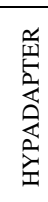 & 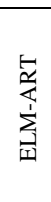 & 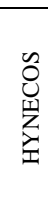 & 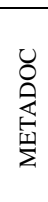 \\
\hline Learner identification & $\bar{X}$ & $\bar{X}$ & $\mathrm{X}$ & - & $\bar{X}$ & $\bar{X}$ & $\mathrm{X}$ & $\bar{X}$ & $\bar{X}$ & - \\
\hline Initialization of the model & $\mathrm{X}$ & $\mathrm{X}$ & - & $\mathrm{X}$ & - & - & $\mathrm{X}$ & - & - & $\mathrm{X}$ \\
\hline Retention of learning states & $\mathrm{X}$ & $\mathrm{X}$ & - & - & $\mathrm{X}$ & - & $\mathrm{X}$ & - & $\mathrm{X}$ & - \\
\hline Update of the model & - & - & - & - & $\mathrm{X}$ & - & $\mathrm{X}$ & - & - & - \\
\hline Inference of other parameters & - & - & $\mathrm{X}$ & - & - & - & - & $\mathrm{X}$ & - & - \\
\hline knowledge modeling & - & - & - & $\mathrm{X}$ & - & - & $\mathrm{X}$ & - & - & - \\
\hline
\end{tabular}

Table 5. Comparative table between the different methodes/approches used in learner modelling in existing AHES

\begin{tabular}{|c|c|c|c|c|c|c|c|c|c|c|}
\hline Learner model modeling techniques/ methods & 起 & 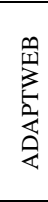 & 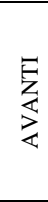 & 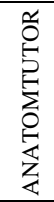 & $\underset{4}{\frac{1}{4}}$ & 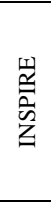 & 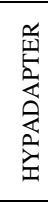 & 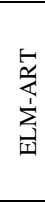 & 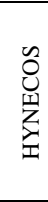 & 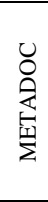 \\
\hline Stereotypes & - & - & $\mathrm{X}$ & $\mathrm{X}$ & - & $\mathrm{X}$ & - & - & $\mathrm{X}$ & $\mathrm{X}$ \\
\hline Overlay Model & $\mathrm{X}$ & - & $\mathrm{X}$ & - & $\mathrm{X}$ & - & $\mathrm{X}$ & $\mathrm{X}$ & - & - \\
\hline Perturbation model & - & - & - & - & $\mathrm{X}$ & - & - & - & - & - \\
\hline Differential model & $\mathrm{X}$ & - & - & - & - & - & - & - & - & - \\
\hline Machine Learning & - & $\mathrm{X}$ & - & - & - & $\mathrm{X}$ & - & $\mathrm{X}$ & - & - \\
\hline Bayesian Networks & - & - & - & - & $\mathrm{X}$ & - & $\mathrm{X}$ & $\mathrm{X}$ & - & - \\
\hline
\end{tabular}

The information stored in a learner model varies between different models and depends on the adaptive elearning system surrounded or often used. To provide as much interoperability for a learner modeling system to be used by multiple systems, it is necessary to agree on the information contained in a learner model. These agreements are represented by standards. There are several standards in the field of user modeling that we will describe in the next chapter.

As a conclusion, it is safe to say that the learner model plays an important role in most hypermedia system and adaptive educational hypermedia systems in particular. We have presented in previous work, a probabilistic approach to manage dynamically the learner model in AHES [20] [21] with an approach that can manage two types of independent and specific information of the domain of the learner, and that can be relevant to the three stages of the construction of the learner model. And we have also presented a process to combine the stereotypes method and Bayesian networks to initialize the learner model in AHES [22] in a probabilistic work frame to respond to the problematic of uncertainty in the learner model.

\section{References}

[1] Kobsa, A., (1993). User modeling: Recent work, prospects and hazards. Human Factors in Information Technology, 10, 111-111.

[2] Brusilovsky, P., (2001). User modeling and user-adapted interaction.

[3] Han, B., (2001). Student modelling and adaptivity in webbased learning systems. Massey University New Zealand.
[4] Zhou, Y., \& Evens, M. W., (1999). A practical student model in an intelligent tutoring system. In Tools with Artificial Intelligence, 1999. Proceedings. 11th IEEE International Conference on (pp. 13-18). IEEE.

[5] De Bra, P., Aerts, A., Berden, B., De Lange, B., Rousseau, B., Santic, T., ... \& Stash, N., (2003, August). AHA! The adaptive hypermedia architecture. In Proceedings of the fourteenth ACM conference on Hypertext and hypermedia (pp. 81-84). ACM.

[6] Warpechowski, M., De Oliveira, J. P. M., Souto, M. A. M., Musa, D. L., Brunetto, M. A. C., de Freitas, V., \& Proença Jr, M. L., (2004). Adaptive hypermedia in the adaptweb environment. In First International Workshop on Engineering the Adaptive Web (EAW) (Vol. 1, pp. 68-73).

[7] Beaumont I., (1994) User modelling in the interactive anatomy tutoring system Anatom-Tutor. User modeling and User-Adapted Interaction, 4: 1994, (p.21-45).

[8] L. Hardman, D.C.A. Bulterman, (1997). Document Model Issues for Hypermedia. In "The Handbook of Multimedia Information Management", edited by W.I. Grosky, R. Jain and R. Mehrotra. Prentice Hall.

[9] Weber, G., \& Brusilovsky, P., (2001). ELM-ART: An adaptive versatile system for Web-based instruction. International Journal of Artificial Intelligence in Education (IJAIED), 12, 351-384.

[10] Papanikolaou, K. A., Grigoriadou, M., Kornilakis, H., \& Magoulas, G. D., (2003). Personalizing the Interaction in a Web-based Educational Hypermedia System: the case of INSPIRE. User modeling and user-adapted interaction, 13(3), 213-267.

[11] M. Niederhausen, S. Karol, U. Aß mann, and K. Meiß ner, "HyperAdapt: Enabling Aspects for XML," in Web 
Engineering, 9th International Conference, ICWE 2009, ser. Lecture Notes in Computer Science, M. Gaedke, M. Grossniklaus, and O. D'1az, Eds. San Sebastian: ' Springer, 2009, pp. 461-464.

[12] Schwabe, D., B. Feijó, and W. Krause: 1990, 'Intelligent Hypertext for Normative Knowledge in Engineering'. In: Rizk, A., N. Streitz, J. André (eds.): Hypertext: Concepts, Systems and Applications. Cambridge: Cambridge University Press.

[13] Boyle, C., \& Encarnacion, A. O., (1998). MetaDoc: an adaptive hypertext reading system. In Adaptive Hypertext and Hypermedia (pp. 71-89). Springer, Dordrecht.

[14] Anouar Tadlaoui, M., Aammou, S., Khaldi, M., \& Carvalho, R. N., (2016). Learner modeling in adaptive educational systems: a comparative study. International Journal of Modern Education and Computer Science, 8(3), 1.

[15] Anouar Tadlaoui, M., Khaldi, M., \& Aammou, S. (2014). Towards a Learning model based on Bayesian Networks. In EDULEARN14 Proceedings (pp. 3185-3193). IATED.

[16] Anouar Tadlaoui, M., (2016). Gestion d'un modèle d'apprenant dans un système éducatif adaptatif basée sur les réseaux bayésiens.

[17] Kay, J., (2001). User modeling for adaptation. User Interfaces for All, Human Factors Series, 271-294.

[18] De Koch, N. P., (2001). Software engineering for adaptive hypermedia systems. Ph. DThesis, Verlag Uni-Druck, Munich.

[19] Anouar Tadlaoui, M., Mohamed, K., \& Souhaib, A., (2014). Towards probabilistic ontology based on Bayesian Networks. International Journal of Software and Web Sciences 1 (10), 102-106.

[20] Anouar Tadlaoui, M., Souhaib, A., \& Mohamed, K., (2015). Learner Modeling Based on Bayesian Networks. In ELearning-Instructional Design, Organizational Strategy and Management. InTech.

[21] Anouar Tadlaoui, M., Carvalho, R. N., \& Khaldi, M., (2017). The initialization of the learner model combining the Bayesian networks and the stereotypes methods. International Journal of Advanced Computer Research, 7(33), 200-21. 